Manuscript ID: AMB-06-13403

\title{
A leuC mutation leading to increased L-lysine production and rel-independent global expression changes in Corynebacterium glutamicum
}

Mikiro Hayashi · Hiroshi Mizoguchi · Junko Ohnishi · Satoshi Mitsuhashi ·

Yoshiyuki Yonetani $\cdot$ Shin-ichi Hashimoto $•$ Masato Ikeda

M. Hayashi $\cdot$ H. Mizoguchi $\cdot$ J. Ohnishi $\cdot$ S. Mitsuhashi $\cdot$ Y. Yonetani

BioFrontier Laboratories, Kyowa Hakko Kogyo Co., Ltd., Machida, Tokyo 194-8533, Japan

S. Hashimoto

Technical Research Laboratories, Kyowa Hakko Kogyo Co., Ltd., Hofu, Yamaguchi 747-8522, Japan

M. Ikeda

Department of Bioscience and Biotechnology, Faculty of Agriculture, Shinshu University, Nagano 399-4598, Japan

e-mail: m_ikeda@shinshu-u.ac.jp

Fax: + 81-265-77-1629 


\begin{abstract}
We previously found by transcriptome analysis that global induction of amino acid-biosynthetic genes occurs in a classically derived industrial L-lysine producer, Corynebacterium glutamicum B-6. Based on this stringent-like transcriptional profile in strain B-6, we analyzed the relevant mutations from among those identified in the genome of the strain, with special attention to the genes that are involved in amino acid biosynthesis and metabolism. Among these mutations, a Gly-456 $\rightarrow$ Asp mutation in the 3-isopropylmalate dehydratase large subunit gene (leuC) was defined as a useful mutation. Introduction of the leuC mutation into a defined L-lysine producer, AHD-2 (hom59 and lysC311), by allelic replacement led to the phenotype of a partial requirement for L-leucine and approximately 14\% increased L-lysine production. Transcriptome analysis revealed that many amino acid-biosynthetic genes including lysC-asd operon were significantly up-regulated in the leuC mutant in a rel-independent manner.
\end{abstract}

\title{
Introduction
}

Amino acid fermentation has grown into a global industry. The leading actors in this technology are regulatory mutants of Corynebacterium glutamicum developed by the conventional technology (Ikeda 2003). Today, the market of L-amino acids is highly competitive and process economics are of primary importance. For cost-effective production, innovation in fermentation technologies has become an urgent need in industry. Also, mechanisms for high-level production are questions of great interest for researchers in this field because the industrial levels of production are still veiled in mystery.

Based on the progress in genomics, we have developed a genome-based approach to create a minimally mutated C. glutamicum strain for efficient production and for understanding of high-level production. By using L-lysine fermentation as a model, we have illustrated that strain development based on the specific incorporation of biotechnologically useful mutations in a robust wild-type strain can substantially improve producer performance (Ohnishi et al. 2002, 2003, 2005; Ikeda et al. 2005).

While verifying the usefulness of this methodology, we found that industrial 
levels of L-lysine production were not attained by our reconstructed strain that carried useful mutations in the relevant L-lysine-biosynthetic pathways (Ikeda et al. 2006), suggesting the presence of other unknown mechanisms for production. To address these, we investigated the transcriptome of industrial L-lysine producer B-6, which was derived by multiple rounds of mutagenesis from wild-type strain ATCC 13032 (Hirao et al. 1989). As the result, we found that many amino acid-biosynthetic genes including the lysC-asd operon, the key genes for L-lysine biosynthesis, were up-regulated in the producer (Hayashi et al. 2006). These findings suggest that a certain global regulatory mechanism is involved in the industrial levels of L-lysine production. Thus, we assume that the global induction of the amino acid-biosynthetic genes might contribute to the increased L-lysine production through increased expression of lysC-asd.

Recent studies have suggested the presence of the (p)ppGpp-dependent stringent control mechanism in the coryneform bacteria, like Escherichia coli (Wehmeier et al. 1998, 2001; Tauch et al. 2001). If so, it is likely that a certain mutation(s) might provoke this stringent response, thus leading to the global induction of the amino acid-biosynthetic genes in strain B-6. Based on the stringent-like transcriptional profile in strain B-6, we attempted to identify the relevant mutations from among those identified in the genome of strain B-6, with special attention to the genes that are involved in amino acid biosynthesis and metabolism. Among these mutations, one in the 3-isopropylmalate dehydratase large subunit gene (leuC) was defined as a useful mutation. Focusing on the beneficial leuC mutation, we describe the metabolic and transcriptome consequence of the mutation in a defined L-lysine producer AHD-2.

\section{Materials and methods}

Bacterial strains and plasmids

The L-lysine-producing strain used for comparative genomic analysis is C. glutamicum B-6 (Hirao et al. 1989) derived by multiple round of mutagenesis from a wild-type strain C. glutamicum ATCC 13032. This production strain has many mutations that lead 
to resistance to L-lysine structural analog, S-(2-aminoethyl)-L-cysteine (AEC), rifampicin, streptomycin, and 6-azauracil. C. glutamicum AHD-2 (Ohnishi et al. 2002), used as a host for evaluation of mutations on the L-leucine-biosynthetic pathway of strain B-6, is a defined L-lysine producer developed by a genome-based approach from the wild-type ATCC 13032. It carries only two useful mutations (hom59 and lysC311) on the relevant terminal pathways, and thus we considered the strain most suitable for a host strain to evaluate the general effect of the leuC mutation on L-lysine production and transcriptome in this organism. Escherichia coli DH5 $\alpha$ was used as a host for cloning of the PCR products. Plasmid pCleuC456 that contained the mutant leuC gene in vector pESB30 (Ohnishi et al. 2002) was used to replace the wild-type chromosomal gene by the mutant gene. Defined chromosomal deletion in the rel gene of C. glutamicum was constructed using plasmid pCdelrel that contained a $1.4 \mathrm{~kb}$ SalI digested apt-rel fragment (Wehmeier et al. 1998) in vector pESB30.

Media and culture conditions

Complete media BY (Katsumata et al. 1984) and minimal medium MM (Ozaki et al. 1985) were used for cultivation of C. glutamicum. Solid plates were made by the addition of Bacto-Agar (Difco) to 1.6\%. When required, kanamycin and L-leucine were added to final concentrations of $25 \mu \mathrm{g} / \mathrm{ml}$ for BY plates and $50 \mu \mathrm{g} / \mathrm{ml}$ for MM plates, respectively. LSS1 and LPG1 media (Ohnishi et al. 2002) were used for second-seed culture and production, respectively, in 5-1 jar fermentors. Cultivations for L-lysine production using 5-1 jar fermentors were carried out as described previously (Ohnishi et al. 2002). For growth of E. coli, LB medium (Sambrook and Russell 2001) was used. Cell growth, glucose consumption, and L-lysine titer were measured as described previously (Ohnishi et al. 2002). L-Lysine titer was determined as L-lysine HCl.

Recombinant DNA techniques

Standard protocols (Sambrook and Russell 2001) were used for the construction, purification and analysis of plasmid DNA, and transformation of E. coli. Chromosomal DNA was extracted from protoplasts of C. glutamicum ATCC 13032 and B-6 by the 
method of Saito and Miura (1963). The protoplasts were prepared by the method of Katsumata et al. (1984). Transformation of C. glutamicum by electroporation was carried out by the method of van der Rest et al. (1999), using a Gene Pulser and Pulse controller (Bio-Rad, USA). PCR was performed with a DNA Thermal Cycler GeneAmp 9700 (Perkin Elmer, USA), using Taq polymerase (Roche, Germany) for routine application, or Pyrobest DNA polymerase (Takara, Japan), when high fidelity was required.

Identification of a mutation in leuC

The leuC gene was PCR amplified from genomic DNA of L-lysine producer B-6. PCR primer sequences were designed as follows.

leuC-F: 5'- CGGTCTAAAACCTTAATACCGCTCT

leuC-R: 5'- AGACCAAGACCTTAAGGGAATCCA

The PCR product was cloned into vector pESB30 to yield plasmid pCleuC456 by the TA cloning method as described previously (Ohnishi et al. 2002). The nucleotide sequence of the cloned leuC gene was then analyzed and compared with the wild-type sequence already available.

Generation of defined mutant ADL-3

The leuC mutation, G456D, on pCleuC456 was introduced into L-lysine producer AHD-2 (hom59 and lysC311) via two recombination events as described previously (Ohnishi et al. 2002). A strain carrying the leuC mutation was designated ADL-3.

Northern blot hybridization and transcriptome analysis

Total RNAs from C. glutamicum were isolated and purified as described previously (Hayashi et al. 2002). For hybridization, aliquots of this RNA were separated on an agarose gel containing $18 \%(\mathrm{v} / \mathrm{v})$ formaldehyde and transferred onto a nylon membrane. The 1266 base full-length DNA fragment of the lys $C$ gene was amplified by PCR and used to prepare a digoxigenin-labelled probe. Labeling of the probe, hybridization, 
washing, and detection were performed using a DIG-High Prime DNA Labeling and Detection Starter Kit II as recommended by the supplier (Roche, Germany). Transcriptome analysis was performed using GeneChip (Affymetrix, USA). Labeling of RNA transcripts, hybridization, and scanning were performed according to the manufacturer's instructions. Gene expression data was analyzed using Microarray Suite 5.0 software (Affymetrix). Changes in expression levels that had a change call of decrease or increase together with a $P$ value of $<0.001$ and a signal ratio of more than 1.5-fold were considered significant. Cultivations for northern blot and transcriptome analysis were performed with 5-1 jar fermentors and total RNAs were prepared from mid-exponential phase cultures. The reproducibility of the measurements was confirmed by duplicate, independent cultures and experiments.

\section{Results}

Identification and characterization of mutation points

Industrial L-lysine producer B-6 is known to exhibit the phenotype of a partial requirement for L-leucine (Hayashi et al. 2006). In addition, our array experiments showed that the leuC and leuD genes encoding the large subunit and the small subunit of 3-isopropylmalate dehydratase, respectively, were about 50-fold up-regulated in strain B-6 as compared to wild-type ATCC 13032 (Hayashi et al. 2006). Considering these observations, it is likely that a certain mutation(s) causes attenuation of L-leucine biosynthesis, which might result in not only release of leuCD from the possible repression mechanism, but also the global induction of the amino acid-biosynthetic genes in strain B-6. Thus, we analyzed the L-leucine-biosynthetic genes of strain B-6 and found a Gly-456 $\rightarrow$ Asp mutation in the leuC gene, an Asp-53 $\rightarrow$ Asn mutation in the $l e u B$ gene encoding 3-isopropylmalate dehydrogenase gene, and a Gly-184 $\rightarrow$ Asp mutation in the ilvE gene encoding branched-chain amino acid aminotransferase. Because there were no other mutations on the L-leucine-biosynthetic pathway in strain B-6, it was expected that any of those three mutations was relevant to the phenotype of a partial requirement for L-leucine in strain B-6. To examine this possibility, each 
mutation was introduced into a defined L-lysine producer, AHD-2 (hom59 and lysC311), by allelic replacement. Growth experiments revealed that only the leuC mutant, designated ADL-3, required L-leucine for maximum growth on MM plates (Fig. 1), finding that the Gly-456 $\rightarrow$ Asp mutation in the leuC gene (designated leuC456) was a mutation leading to a partial requirement for L-leucine for growth.

Evaluation of the leuC456 mutation on L-lysine production and transcriptome

L-Lysine production by strain ADL-3 was investigated using 5-1 jar fermentors, with strain AHD-2 as control. Figure 2a shows the profiles obtained in fed-batch fermentor cultivation with glucose medium to which sugar was added at an initial concentration of $5 \%$, with a subsequent increase to $25 \%$. The growth profile of strain ADL-3 was slightly different from strain AHD-2 on the point that strain ADL-3 showed somewhat limited growth in the exponential phase. In case of strain ADL-3, it took about 9 hours to consume the 5\% sugar initially added to the medium, while it took about 7 hours in case of strain AHD-2. The growth level $\left(\mathrm{OD}_{660}\right)$ of strain ADL-3 was also limited as compared to strain AHD-2 at the point where the 5\% sugar was used up. However, during the feeding of sugar to the medium, the growth of strain ADL-3 reached close levels of that of strain AHD-2, and its final $\mathrm{OD}_{660}$ became equivalent to that of strain AHD-2 as shown in Fig. 2a. Under such growth conditions, strain ADL-3 accumulated approximately 14\% higher L-lysine (83 g/1) than strain AHD-2 (73 g/l), indicating that the leuC456 mutation was relevant to L-lysine production. When an excess amount of L-leucine was added into the medium for strain ADL-3, such differences in both growth kinetics and production were not observed between the two strains (data not shown), showing that in the culture conditions used, strain ADL-3 was under L-leucine limitation, which was responsible for the higher performance of strain ADL-3.

To elucidate underlying mechanism for the increased production by the introduction of leuC456, strain ADL-3 was analyzed using GeneChip. Total RNAs were prepared from mid-exponential phase cultures (arrows in Fig. 2). The correlation coefficient of the signal intensity between strains AHD-2 and ADL-3 was 0.98, indicating no drastic changes in the gene expression profiles between the two strains. Actually, we could not observe significant changes in the central metabolic pathway 
genes between the two strains. However, many amino acid-biosynthetic genes were significantly up-regulated in strain ADL-3 (Table 1). Noteworthy were the leuCD genes, which were exceptionally up-regulated as much as about 25 -fold, suggesting release from the possible repression mechanism as a consequence of attenuation of L-leucine biosynthesis as observed in strain B-6 (Hayashi et al. 2006). In addition, lysC-asd was about 1.5- to 2-fold up-regulated in strain ADL-3 as observed in strain B-6. Though the expression levels varied relatively small in the GeneChip experiment, increased transcript level of lysC-asd in strain ADL-3 was confirmed clearly by northern blot hybridization (Fig. 3). Increased transcript levels of serA, aroBKC, and trpEDCBA were also observed in strain ADL-3 as in the case of strain B-6. However, their expression levels varied much smaller ranges than those of strain B-6 (Hayashi et al. 2006). We confirmed that the differential expression measurement obtained were reproducible by duplicate, independent cultures and experiments.

Effects of rel disruption on L-lysine production and transcriptome

It was reported that a C. glutamicum mutant carrying a defined deletion in the rel gene did not accumulate (p)ppGpp and thereby failed to give rise to the stringent response upon amino acid starvation (Wehmmeier et al. 1998). On the basis of the findings, defined chromosomal deletions in the rel genes of strains AHD-2 and ADL-3 were constructed using plasmid pCdelrel to investigate the relation between the effect of leuC456 and the stringent control mechanism. The resulting rel-deleted strains of AHD-2 and ADL-3, designated AHD-2 $\Delta$ rel and ADL-3 $\Delta$ rel, respectively, were then tested for L-lysine production in 5-1 jar fermentors (Fig. 2b). Interestingly, the growth of both strains, AHD-2 $\Delta r e l$ and ADL-3 $\Delta r e l$, was improved and L-lysine titers were reduced as compared to the rel-positive backgrounds. When growth and L-lysine production were compared between strains AHD-2 $\Delta r e l$ and ADL-3 $\Delta r e l$, the latter strain showed slightly limited growth and significantly higher L-lysine production than the former strain, as in the rel-positive backgrounds.

We next examined the effect of the leuC456 mutation on the transcriptome in the rel-negative backgrounds. If the stringent control mechanism is responsible for the global gene expression changes caused by leuC456, no changes of gene expression will 
be observed in the rel-negative backgrounds. When total RNAs from mid-exponential phase cultures of 5-1 jar fermentors were used to study differential transcription profiles between strains AHD-2 $\Delta r e l$ and ADL-3 $\Delta r e l$, we still observed up-regulation of the amino acid-biosynthetic genes in strain ADL-3 $\Delta$ rel as in the case of the rel-positive backgrounds (Table 1). This result suggests that the observed up-regulation of the amino acid-biosynthetic genes by leuC456 is independent of the stringent control mechanism.

\section{Discussion}

It is known that some mutants classically derived from coryneform bacteria for L-lysine production are L-leucine auxotrophs (Tosaka et al. 1978). These strains accumulate considerable amounts of L-lysine, provided that L-leucine-limiting conditions are established (Patek et al. 1994). It is also reported that L-leucine-auxotrophic mutation leads to increased L-lysine yield in C. glutamicum (Schrumpf et al. 1992). As illustrated by these work, it has been known for long time that L-leucine auxotrophy has positive influence on L-lysine production in the coryneform bacteria.

To date, there have been two possible explanations for the positive effect of L-leucine limitation on L-lysine production. One is release from the L-leucine-dependent repression of the dapA gene encoding dihydrodipicolinate synthase which is the first enzyme of the L-lysine-biosynthetic pathway (Tosaka et al. 1978). The other is the decreased biomass which might allow increased precursor supply from central metabolism into the L-lysine-biosynthetic pathway through minimizing the loss of carbon required for cell growth (Patek et al. 1994). However, these two hypotheses seem not to be enough to reasonably explain our case, because we could not observe any significant up-regulation of $\operatorname{dap} A$ (data not shown) and also any significant decrease in the final growth level in strain ADL-3 (Fig. 2a), as compared to parental strain AHD-2. Instead, we newly found in this work that the transcript levels of many amino acid-biosynthetic genes including the lysC-asd operon were significantly increased in strain ADL-3 (Fig. 3, Table 1). This observation is noteworthy because nothing has been known about the effect of L-leucine limitation on transcriptome in this bacterium while L-leucine-auxotrophic C. glutamicum strains were analyzed so far from the viewpoint of 
alteration of enzyme activities (Schrumpf et al. 1992; Patek et al. 1994). On the basis of our findings, we propose additional hypothesis as follows: L-leucine limitation caused by the leuC456 mutation might give rise to the global induction of the amino acid-biosynthetic genes through a certain regulatory mechanism, which likely contribute to increased L-lysine production through increased expression of lysC-asd. This hypothesis is also supported by our previous observation that duplication of the desensitized lysC-asd operon on the chromosome had a positive effect on L-lysine production in strain AHD-2 (data not shown).

In addition to lysC-asd, many amino acid-biosynthetic genes were up-regulated in strain ADL-3 (Table 1), as in the case of industrial strain B-6 (Hayashi et al. 2006). Though such a kind of global regulation has not so far been known in C. glutamicum, it reminded us of (p)ppGpp-dependent stringent control mechanism known in E. coli and other bacteria (Cashel et al. 1996). However, our transcriptome analysis showed that the up-regulation of the amino acid-biosynthetic genes observed in strain ADL-3 remained almost unchanged upon the disruption of the rel gene. This result indicates that the up-regulation is independent of rel and thus is not involved in the stringent control mechanism. Unexpectedly, the growth of the rel-deleted strains was improved as compared to the rel-positive strains in 5-1 jar fermentations (Fig. 2). It has been reported that a C. glutamicum rel mutant is unable to reduce the expression of the rrn gene encoding rRNA upon amino acid starvation (Tauch et al. 2001). Because rRNA transcription is the rate-limiting step in ribosome synthesis, it is likely that the deletion of the rel gene causes relatively increased protein synthesis by keeping the expression of rRNA high, which might result in the growth improvement of the rel-deleted strains.

Another candidate of such regulatory mechanisms could be an Lrp-type transcription factor. The Lrp is a global regulator in E. coli, affecting the expressions of many genes and operons including the catabolism and anabolism of amino acids (Newman et al. 1996). In C. glutamicum, an Lrp-type transcription factor is located adjacent to the brnFE operon which codes for L-isoleucine- and L-methionine-export protein and is known to be responsible for regulation of the operon (Kennerknecht et al. 2002; Trötschel et al. 2005). We evaluated the effect of deletion of Irp on both L-lysine production and transcriptome. However, no significant difference was observed (data not shown). In this context, it has been reported that most Irp homologues that were 
characterized experimentally have a specific role rather than a global role (Brinkman et al. 2003). Also in case of C. glutamicum, Lrp is likely to be responsible only for the regulation of the brnFE operon. Putting all these results together, the global gene expression changes observed in the leuC456 mutant seems to depend on other unknown transcriptional regulators.

In this study, we showed that the leuC456 mutation led to increased L-lysine production and rel-independent global gene expression changes. However, considering that the induction levels were much smaller than industrial strain B-6, only leuC456 is not sufficient to explain the transcriptome profile observed for strain B-6. Presumably, other uncharacterized mutations might be involved in the profile and also the industrial levels of L-lysine production. Further genomic and transcriptome analysis of the industrial strain will provide guidance for exploration of the relevant mutation(s).

Acknowledgements The authors thank Drs. S. Teshiba and A. Ozaki for encouraging support of our work, and also T. Abe, Y. Adachi, S. Nakagawa, S. Koizumi, and M. Yagasaki for useful discussions, and A. Yamane and K. Ishimaru for their excellent technical assistances.

\section{References}

Brinkman AB, Ettema TJ, de Vos WM, van der Oost J (2003) The Lrp family of transcriptional regulators. Molecular Micorobiology 48:287-294

Cashel M, Gentry DR, Hernandez, VJ, Vinella D (1996) The stringent response. In: Neidhardt F (ed) Escherichia coli and Salmonella : Cellular and Molecular Biology. American Society for Microbiology Press, Washington, D. C., pp. 1458-1496

Hayashi M, Mizoguchi H, Shiraishi N, Obayashi M, Nakagawa S, Imai J, Watanabe S, Ota T, Ikeda M (2002) Transcriptome analysis of acetate metabolism in Corynebacterium glutamicum using a newly developed metabolic array. Biosci Biotechnol Biochem 66:1337-1344

Hayashi M, Ohnishi J, Mitsuhashi S, Yonetani Y, Hashimoto S, Ikeda M (2006)

Transcriptome analysis reveals global expression changes in an industrial L-lysine 
producer of Corynebacterium glutamicum. Biosci Biotechnol Biochem 70:546-550

Hirao T, Nakano T, Azuma T, Sugimoto M, Nakanishi T (1989) L-Lysine production in continuous culture of an L-lysine hyperproducing mutant of Corynebacterium glutamicum. Appl Microbiol Biotechnol 32:269-273

Ikeda M (2003) Amino acid production processes. In: Faurie R, Thommel J (eds), Adv Biochem Eng Biotechnol vol. 79. Microbial production of L-amino acids. Springer-Verlag, Berlin, Heidelberg, pp 1-35

Ikeda M, Ohnishi J, Hayashi M, Mitsuhashi S (2006) A genome-based approach to create a minimally mutated Corynebacterium glutamicum strain for efficient L-lysine production. J Ind Microbiol Biotechnol in press

Ikeda M, Ohnishi J, Mitsuhashi S (2005) Genome breeding of an amino acid-producing Corynebacterium glutamicum Mutant. In: Barredo JLS (ed) Microbial processes and products. Humana Press, Totowa, pp 179-189

Katsumata R, Ozaki A, Oka T, Furuya (1984) A Protoplast transformation of glutamate-producing bacteria with plasmid DNA. J Bacteriol 159:306-311

Kennerknecht N, Sahm H, Yen MR, Patek M, Saier MH Jr, Eggeling L (2002) Export of L-Isoleucine from Corynebacterium glutamicum : a Two-Gene-Encoded Member of a New Translocator Family. J Bacteriol 184:3947-3956

Newman EB, Lin RT, D'ari R (1996) The Leucine/Lrp regulon. In: Neidhardt F (ed) Escherichia coli and Salmonella : Cellular and Molecular Biology. American Society for Microbiology Press, Washington, D. C., pp. 1513-1525

Ohnishi J, Hayashi M, Mitsuhashi S, Ikeda M (2003) Efficient $40^{\circ} \mathrm{C}$ fermentation of L-lysine by a new Corynebacterium glutamicum mutant developed by genome breeding. Appl Microbiol Biotechnol 62:69-75

Ohnishi J, Katahira R, Mitsuhashi S, Kakita S, Ikeda M (2005) A novel gnd mutation leading to increased L-lysine production in Corynebacterium glutamicum. FEMS Microbiol Lett 242:265-274

Ohnishi J, Mitsuhashi S, Hayashi M, Ando S, Yokoi H, Ochiai K, Ikeda M (2002) A novel methodology employing Corynebacterium glutamicum genome information to generate a new L-lysine-producing mutant. Appl Microbiol Biotechnol $58: 217-223$

Ozaki A, Katsumata R, Oka T, Furuya A (1985) Cloning of the genes concerned in 
phenylalanine biosynthesis in Corynebacterium glutamicum and its application to breeding of a phenylalanine producing strain. Agric Biol Chem 49:2925-2930

Patek M, Krumbach K, Eggeling L, Sahm H (1994) Leucine synthesis in Corynebacterium glutamicum: enzyme activities, structure of leuA, and effect of leuA inactivation on lysine synthesis. Appl Environ Microbiol 60:133-140

Rest ME van der, Lange C, Molenaar D (1999) A heat shock following electroporation of Corynebacterium glutamicum with xenogenein plasmid DNA. Appl Microbiol Biotechnol 52:541-545

Saito H, Miura K (1963) Preparation of transforming deoxyribonucleic acid by phenol treatment. Biochem Biophys Acta 72: 619-629

Sambrook J, Russell DW (2001) Molecular cloning: a laboratory manyal, 3rd edn. Cold Spring Harbor Laboratory Press, Cold Spring Harbor, N.Y.

Schrumpf B, Eggeling L, Sahm H (1992) Isolation and prominent characteristics of an L-lysine hyperproducing strain of Corynebacterium glutamicum. Appl Microbiol Biotechnol 37:566-571

Tauch A, Wehmeier L, Gotker S, Pühler A, Kalinowski, J. (2001) Relaxed rrn expression and amino acid requirement of a Corynebacterium glutamicum rel mutant defective in (p)ppGpp metabolism. FEMS Microbiol Lett 201:53-58

Tosaka O, Hirakawa H, Takinami K, Hirose Y (1978) Regulation of lysine biosynthesis by leucine in Brevibacterium lactofermentum. Agric Biol Chem 42:1501-1506

Trötschel C, Deutenberg D, Bathe B, Burkovski A, Krämer R (2005) Characterization of Methionine Export in Corynebacterium glutamicum. J Bacteriol 187:3786-3794

Wehmeier L, Schäfer A, Burkovski A, Krämer R, Mechold U, Malke H, Pühler A, Kalinowski J (1998) The role of the Corynebacterium glutamicum rel gene in (p)ppGpp metabolism. Microbiology 144:1853-1862

Wehmeier L, Brockmann-Gretza O, Pisabarro A, Tauch A, Pühler A Martin J F, Kalinowski J. (2001) A Corynebacterium glutamicum mutant with a defined deletion within the rplK gene is impaired in (p)ppGpp accumulation upon amino acid starvation. Microbiology 147: 691-700 


\section{Figure legends}

Fig.1 Growth of strains AHD-2 and ADL-3 under minimal medium (a) and minimal medium supplemented with $50 \mu \mathrm{g}$ L-leucine per $\mathrm{ml}(\mathrm{b})$. The plates were incubated at $30^{\circ} \mathrm{C}$ for 3 days

Fig.2 L-Lysine fermentation by strains AHD-2 (closed circles) and ADL-3 (open circles) (a) and strains AHD-2 $\Delta$ rel (closed triangles) and ADL-3 $\Delta r e l$ (open triangles) (b). The results represent mean values from three independent cultures. Individual data of biomass and L-lysine concentrations did not derive from the mean by more than $3 \%$. The arrows indicate the points at which samples were withdrawn for transcriptional analysis

Fig. 3 Northern blot analysis of lysC-asd transcript levels in strains AHD-2 (Lane 1) and ADL-3 (Lane 2). The arrow indicates the hybridizing band corresponding to the expected size of the $2.7 \mathrm{~kb}$ lys $C$-asd transcript. The numbers on the left indicate the sizes of RNA Molecular Weight Marker I, digoxigenin-labelled (Roche) 
Table 1. Effect of the leuC456 mutation on gene expressions of the amino acidbiosynthetic genes. Each value indicates the ratio of the gene expression level in strain ADL-3 compared to that in strain AHD-2

\begin{tabular}{|c|c|c|c|c|}
\hline $\mathrm{NCBI}$ & Gene & Anotation & $\mathrm{rel}^{+}$ & $\mathrm{rel}^{-}$ \\
\hline $\mathrm{NCg10247}$ & lys $C$ & Aspartate kinase alpha chain & 1.9 & 1.6 \\
\hline $\mathrm{NCg} 10248$ & asd & Aspartate-semialdehyde dehydrogenase & 1.5 & 1.5 \\
\hline NCg11235 & $\operatorname{ser} A$ & Phosphoglycerate dehydrogenase & 2.9 & 3.9 \\
\hline $\mathrm{NCg11237}$ & $\operatorname{leu} B$ & 3-Isopropylmalate dehydrogenase & 2.0 & 1.9 \\
\hline $\mathrm{NCgl1262}$ & leuC & 3-Isopropylmalate dehydratase large subunit & 28.2 & 29.4 \\
\hline $\mathrm{NCgl1263}$ & leuD & 3-Isopropylmalate dehydratase small subunit & 25.6 & 23.9 \\
\hline NCg11559 & $\operatorname{aroB}$ & 3-Dehydroquinate synthase & 1.5 & 1.4 \\
\hline NCg11560 & $\operatorname{aroK}$ & Shikimate kinase & 1.9 & 1.5 \\
\hline NCg11561 & aroC & Chorismate synthase & 2.4 & 1.8 \\
\hline $\mathrm{NCg} 12139$ & $\operatorname{thr} C$ & Threonine synthase & 2.5 & 2.2 \\
\hline $\mathrm{NCgl} 2360$ & metB & Cystathionine gamma-synthase & 1.5 & 0.9 \\
\hline $\mathrm{NCg} 12927$ & $\operatorname{trp} E$ & Anthranilate synthase component I & 1.9 & 2.1 \\
\hline NCg12929 & $\operatorname{trpD}$ & Anthranilate phosphoribosyltransferase & 1.7 & 1.9 \\
\hline $\mathrm{NCg} 12930$ & $\operatorname{trpC}$ & Indole-3-glycerol phosphate synthase & 1.8 & 2.1 \\
\hline $\mathrm{NCg} 12931$ & $\operatorname{trp} B$ & Tryptophan synthase beta chain & 1.6 & 1.6 \\
\hline $\mathrm{NCgl} 2932$ & $\operatorname{trp} A$ & Tryptophan synthase alpha chain & 1.6 & 1.6 \\
\hline
\end{tabular}




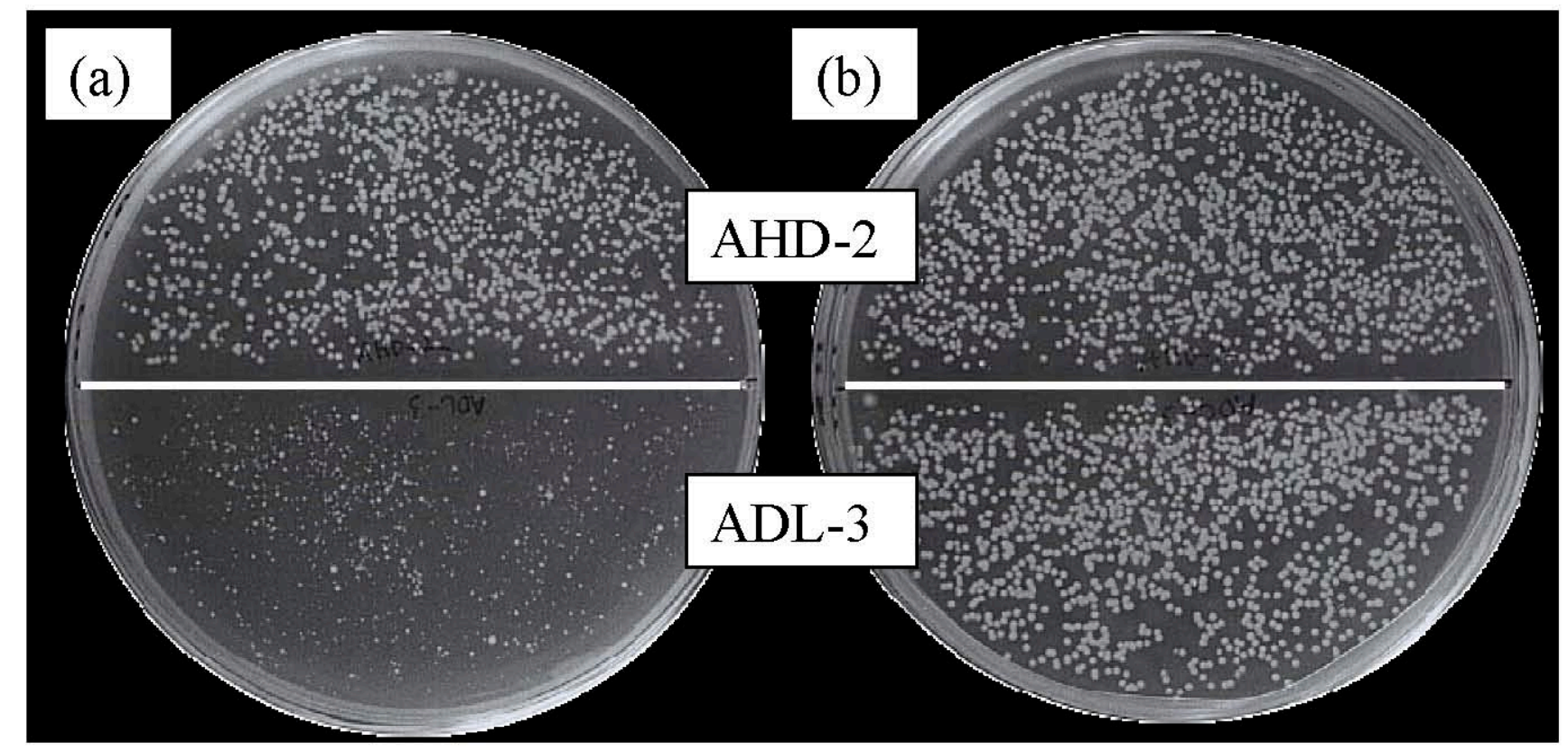

Fig.1 


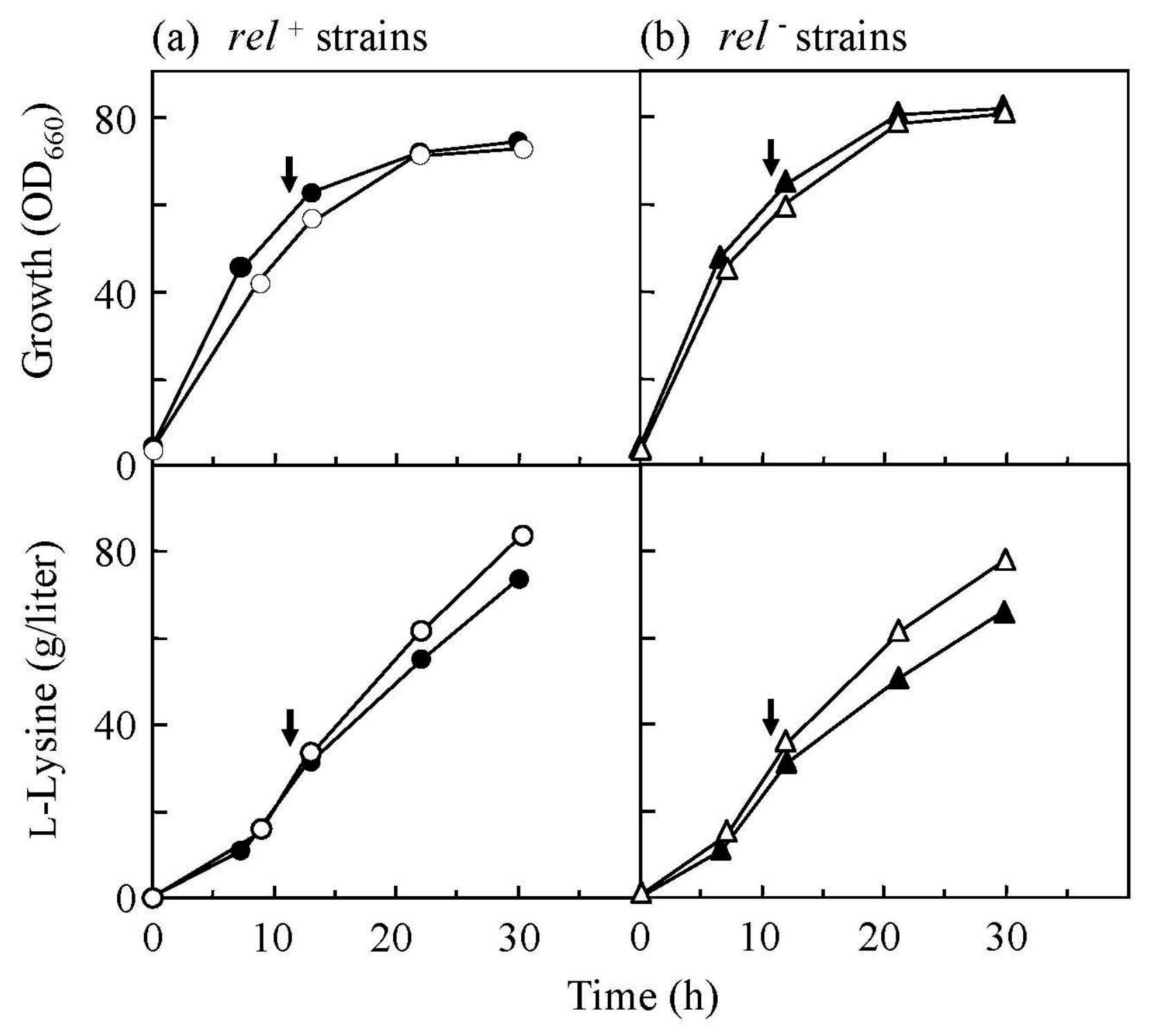

Fig.2 


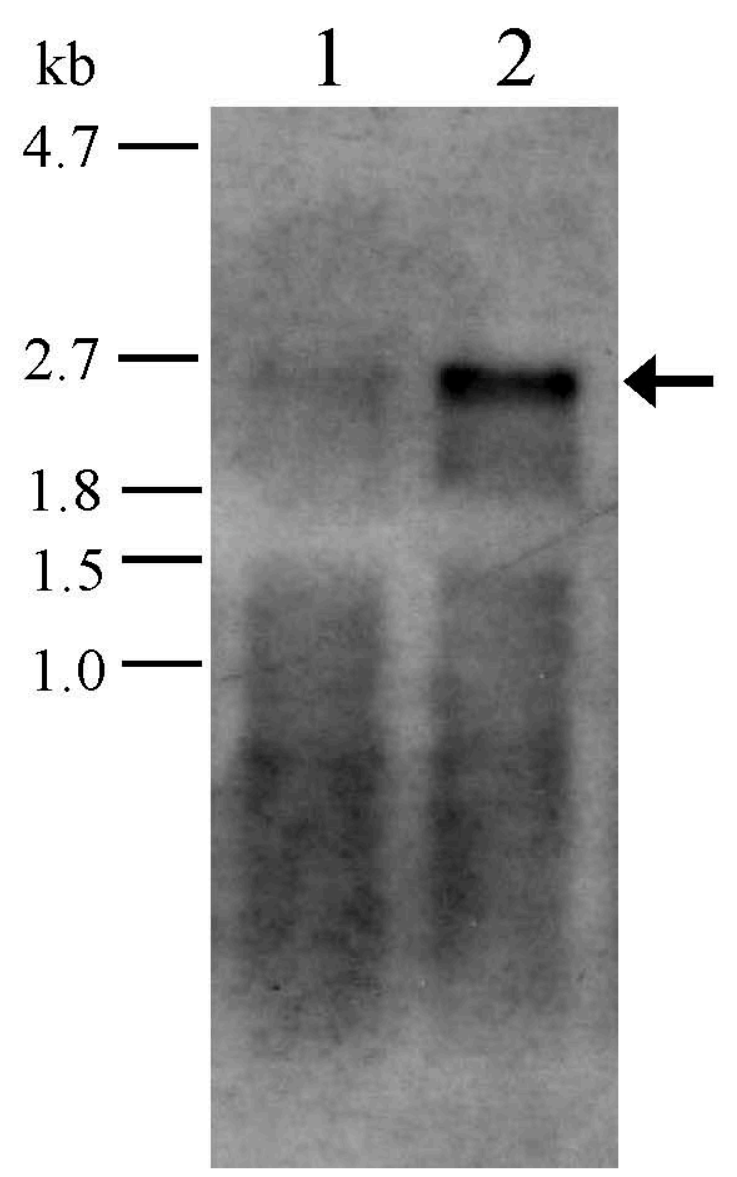

Fig. 3 